\title{
Overweight is associated with better one-year survival in elderly patients after cardiac surgery: a retrospective analysis of the MIMIC-III database
}

\author{
Yiran Zhang^, Qi Zheng, Xiaoyi Dai, Xingjie Xu, Liang $\mathrm{Ma}^{\wedge}$ \\ Department of Cardiovascular Surgery, First Affiliated Hospital, College of Medicine, Zhejiang University, Hangzhou, China \\ Contributions: (I) Conception and design: Y Zhang, L Ma; (II) Administrative support: L Ma; (III) Provision of study materials or patients: Y Zhang, \\ L Ma; (IV) Collection and assembly of data: Y Zhang, Q Zheng, X Dai, X Xu; (V) Data analysis and interpretation: Y Zhang, L Ma; (VI) Manuscript \\ writing: All authors; (VII) Final approval of manuscript: All authors. \\ Correspondence to: Liang Ma, MD. Department of Cardiovascular Surgery, First Affiliated Hospital, College of Medicine, Zhejiang University, \\ Hangzhou 310003, China. Email: m11402@zju.edu.cn.
}

Background: The relationship of body mass index (BMI) to short- and long-term outcomes after cardiac surgery remains controversial, and the dose-response relationship between BMI and mortality in patients receiving cardiac surgery is unclear. Furthermore, the influence of age, concomitant disease, and types of surgery on the prognostic role of BMI has yet to be determined.

Methods: A retrospective cohort study with 6,473 adult patients receiving cardiac surgery was conducted using the Multiparameter Intelligent Monitoring in Intensive Care (MIMIC-III) database. Multivariate Cox proportional hazard analysis and multivariate logistic regression analysis were used to assess the association of BMI with 1-year and in-hospital mortality. Restricted cubic regression splines were used to evaluate the effect of BMI as a continuous variable and to determine appropriate cut points. Subgroup analyses were performed based on age, hypertension and types of surgery.

Results: The baseline characteristics of patients differed between BMI categories. On multivariable analysis, overweight patients (BMI $25-30 \mathrm{~kg} / \mathrm{m}^{2}$ ) had a lower 1-year mortality [hazard ratio $(\mathrm{HR})=0.660$, $95 \%$ confidence interval $(\mathrm{CI}): 0.516-0.843, \mathrm{P}=0.001]$ when compared with normal weight patients (BMI $18.5-25 \mathrm{~kg} / \mathrm{m}^{2}$ ). For patients with $\mathrm{BMI}<30 \mathrm{~kg} / \mathrm{m}^{2}$, each $1 \mathrm{~kg} / \mathrm{m}^{2} \mathrm{BMI}$ increase was independently associated with a significant decrease in the 1-year mortality risk ( $\mathrm{HR}=0.936,95 \% \mathrm{CI}$ : 0.899-0.975, $\mathrm{P}=0.002$ ), while in patients with $\mathrm{BMI} \geq 30 \mathrm{~kg} / \mathrm{m}^{2}$, an increase in BMI did not increase the 1 -year mortality risk (HR $=1.032,95 \%$ CI: 0.998-1.067, $\mathrm{P}=0.064)$. Subgroup analyses suggested the protective effect of overweight on post-cardiac surgery survival was confined to patients with advanced age (>60 years), hypertension and those undergoing isolated coronary artery bypass grafting (CABG).

Conclusions: Overweight was associated with better 1-year survival in patients after cardiac surgery when compared to normal weight. The protective effect of overweight on post-cardiac surgery survival was confined to elderly patients ( $>60$ years).

Keywords: Body mass index; cardiac surgery; mortality; Multiparameter Intelligent Monitoring in Intensive Care database (MIMIC-III database)

Submitted Sep 04, 2020. Accepted for publication Nov 20, 2020.

doi: $10.21037 /$ jtd-20-2824

View this article at: http://dx.doi.org/10.21037/jtd-20-2824

^ ORCID: Yiran Zhang, 0000-0003-1681-9363, Liang Ma, 0000-0001-8054-4582. 


\section{Introduction}

The prevalence of obesity [body mass index (BMI) $\geq 30 \mathrm{~kg} / \mathrm{m}^{2}$ ] and overweight (BMI $25-30 \mathrm{~kg} / \mathrm{m}^{2}$ ) has risen dramatically during the past four decades (1). Obesity adversely affects cardiovascular hemodynamics, structure and function, and increases the risk of most cardiovascular disease (2). Meanwhile, a so called "obesity paradox" has been noted in various studies, suggesting that while obesity has been associated with higher all-cause mortality, overweight has been associated with lower all-cause mortality (3). It has also been shown that obesity could play a protective role in acute coronary syndromes (4) and heart failure (5).

The relationship between body mass index (BMI) and the prognosis of cardiovascular surgery has been reported in several studies. A large nationwide cohort study reported obesity to be associated with lower in-hospital mortality after cardiac surgery (6), and a recent meta-analysis suggested a U-shaped relationship between BMI and allcause mortality following cardiac surgery (7). However, some recent cohort studies have failed to find a relationship between BMI and early/long-term outcome after cardiac surgery $(8,9)$. The protective effect of obesity might be explained by potential mechanisms such as adipokines secretion, reducing oxidative stress and inflammation, and lowering levels of B-type natriuretic peptide (10). While some suggested that the obese paradox might rather be a product of selection bias or confounding bias, and not an observation of a real phenomenon (11).

Thus, the relationship of BMI with short- and longterm outcomes after cardiac surgery remains controversial, and the dose-response relationship between BMI and mortality in patients receiving cardiac surgery is unclear. Moreover, the influences of age, concomitant disease, and types of surgery on the prognostic role of BMI has yet to be determined. To address these issues in depth, we used detailed clinical data obtained from the Multiparameter Intelligent Monitoring in Intensive Care (MIMIC-III) database (12) to investigate the relationship of BMI with in-hospital and 1-year outcomes after cardiac surgery. We present the following article in accordance with the STROBE reporting checklist (available at http://dx.doi. org/10.21037/jtd-20-2824).

\section{Methods}

\section{Data source}

Study data was extracted from the Multiparameter
Intelligent Monitoring in Intensive Care III (MIMIC-III) database version 1.4 (12), which is an open-access database comprising the clinical data of over 40 thousand patients admitted to ICUs at the Beth Israel Deaconess Medical Center (Boston, MA, USA) from 2001 to 2012. The study was conducted in accordance with the Declaration of Helsinki (as revised in 2013).

\section{Study population}

All patients receiving open-heart surgery under extracorporeal circulation (ICD-9 code: 3961 ) were initially screened. Patients older than 18 years old and with weight and height records were included in the study. Patients were then divided into four groups according to the World Health Organization (WHO) BMI classifications: underweight (BMI $<18.5 \mathrm{~kg} / \mathrm{m}^{2}$ ), normal weight (BMI 18.5$25 \mathrm{~kg} / \mathrm{m}^{2}$ ), overweight (BMI $25-30 \mathrm{~kg} / \mathrm{m}^{2}$ ), and obese (BMI $\geq 30 \mathrm{~kg} / \mathrm{m}^{2}$ ).

\section{Data extraction}

Data extraction was executed with structure query language (SQL) in PostgreSQL (v12.2; PostgreSQL Global Development Group). The following clinical variables were extracted: age, sex, weight, height, ethnicity, medical history [hypertension, diabetes, alcohol abuse, smoker, chronic kidney disease (CKD), chronic obstructive pulmonary disease (COPD), obstructive sleep apnea (OSA), peripheral vascular disease], admission type (elective or emergency/ urgent), type of cardiovascular surgery [valve surgery only, coronary artery bypass grafting (CABG) only, CABG + valve surgery, aortic surgery], vital signs within 24 hours after ICU admission [heart rate, systolic blood pressure, diastolic blood pressure, respiratory rate, oxygen saturation $\left(\mathrm{SpO}_{2}\right)$, and blood glucose), laboratory tests within 24 hours after ICU admission (serum creatinine, white blood cell count (WBC), hemoglobin, platelet, partial thromboplastin time (PT), and activated partial thromboplastin time (APTT)], and the Simplified Acute Physiology Score II (SAPS-II) after ICU admission. When variables were measured more than once, the average of the highest and lowest values was taken.

\section{Outcomes}

The primary outcomes were the in-hospital and 1-year mortality. The secondary outcomes included ventilation 


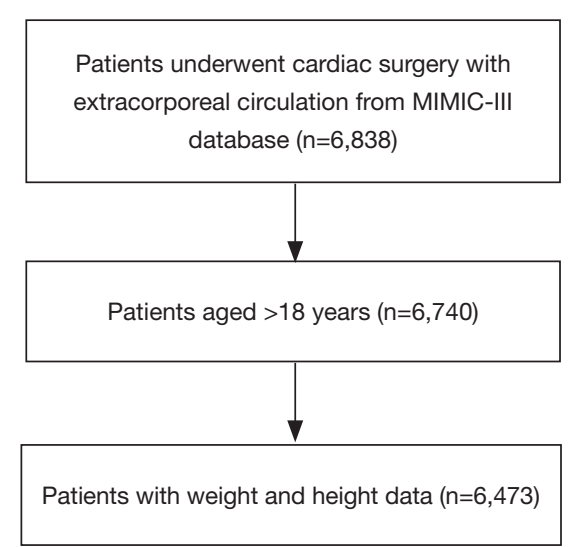

Figure 1 Flowchart of study cohort selection. MIMIC-III, Multiparameter Intelligent Monitoring in Intensive Care.

duration, the time to weaning from vasopressors, length of ICU stays, length of hospital stays, and postoperative continuous renal replacement therapy (CRRT).

\section{Statistical analysis}

Continuous variables were checked for normality, which showed that all the continuous variables in this study were non-normally distributed. Thus, these nonnormally distributed variables were reported as medians with interquartile ranges (IQRs), and compared by the Kruskal-Wallis test. Categorical variables are presented as numbers and proportions, and were compared using the Pearson's chi-square or Fisher's exact test. Multivariate Cox proportional hazard analysis was used to analyze factors related to 1-year mortality with hazard ratios (HRs) and $95 \%$ confidence interval (CI). Multivariate logistic regression analysis was used to identify factors related to in-hospital mortality with odds ratios (ORs) and 95\% CI. Kaplan-Meier survival curves among the four BMI categories were constructed and compared using the logrank test. BMI was also analyzed as a continuous variable. Restricted cubic splines were used to explore the nonlinear association between BMI and 1-year mortality. An optimal BMI cut point was determined to be $30 \mathrm{~kg} / \mathrm{m}^{2}$ and was used as a knot for linear splines. The patient population was then divided into two subgroups (BMI $<30 \mathrm{~kg} / \mathrm{m}^{2}$ and BMI $\geq 30 \mathrm{~kg} / \mathrm{m}^{2}$ ) according to the BMI cut point, and the adjusted HRs with $95 \%$ CIs associated with an increase in BMI by $1 \mathrm{~kg} / \mathrm{m}^{2}$ in these two subgroups are reported respectively. Subgroup analyses were performed based on age, hypertension and types of surgery to determine their confounding influences. A case-control matched analysis was also performed to investigate the confounding influences of age and sex on the study results. Overweight or obese patients were matched 1:1 with normal/underweight patients according to age ( \pm 2 years) and sex. Kaplan-Meier survival curves and subgroup analysis were analyzed again in the matched cohorts. All statistical analyses were processed using SPSS 25.0 software (SPSS Inc., Chicago, IL, USA) and $\mathrm{R}$ programming language (version 4.0.0). All $\mathrm{P}$ values of $<0.05$ were considered statistically significant.

\section{Results}

\section{Patient characteristics}

The MIMIC-III database contains the data of 6,838 patients who receive open-heart surgery under extracorporeal circulation, 6,473 of whom met the inclusion criteria for this study (Figure 1).

The proportions of patients by BMI category were as follows: underweight $\left(\mathrm{BMI}<18.5 \mathrm{~kg} / \mathrm{m}^{2}\right), 1.2 \%(\mathrm{n}=78)$; normal weight (BMI $\left.18.5-25 \mathrm{~kg} / \mathrm{m}^{2}\right), 26.4 \%(\mathrm{n}=1,708)$; overweight (BMI $\left.25-30 \mathrm{~kg} / \mathrm{m}^{2}\right), 38.5 \%(\mathrm{n}=2,495)$; and obese $\left(\mathrm{BMI} \geq 30 \mathrm{~kg} / \mathrm{m}^{2}\right), 33.9 \%(\mathrm{n}=2,192)$. The baseline characteristics of patients are seen in Table 1. Patients with overweight (median age, 68 years) or obesity (median age, 65 years) were younger than normal weight patients (median age, 70). The proportion of male was higher in the overweight group (74.2\%) compared with the normal weight group (63.9\%), but was lower in the underweight group (34.6\%). Meanwhile, the proportion of Asian patients in the overweight group (1.4\%) and obese group (0.5\%) was lower compared to the normal weight group (3.6\%). Overweight and obese patients had a higher prevalence of hypertension $(71.0 \%$ and $75.0 \%$ vs. $62.6 \%)$ and diabetes (28.3\% and $44.1 \%$ vs. $22.7 \%$ ) than normal weight patients. The prevalence of COPD was lowest in the overweight group (12.2\%). Obese patients had the highest proportion of OSA $(7.9 \%)$, and the lowest proportion of peripheral vascular disease $(16.1 \%)$. The proportions of valve surgery in the overweight group (19.6\%) and obese group (18.1\%) were lower compared to the normal weight group (23.8\%), while the proportions of CABG in the overweight group (55.2\%) and obese group (59.7\%) were higher compared to the normal weight group (46.7\%). Overweight and obese patients had higher diastolic blood pressure, respiratory rate, blood glucose, serum creatinine, hemoglobin, PT, and lower $\mathrm{SpO}_{2}$, and APTT compared with normal weight 
Table 1 Baseline characteristics of 6,473 study patients by BMI category

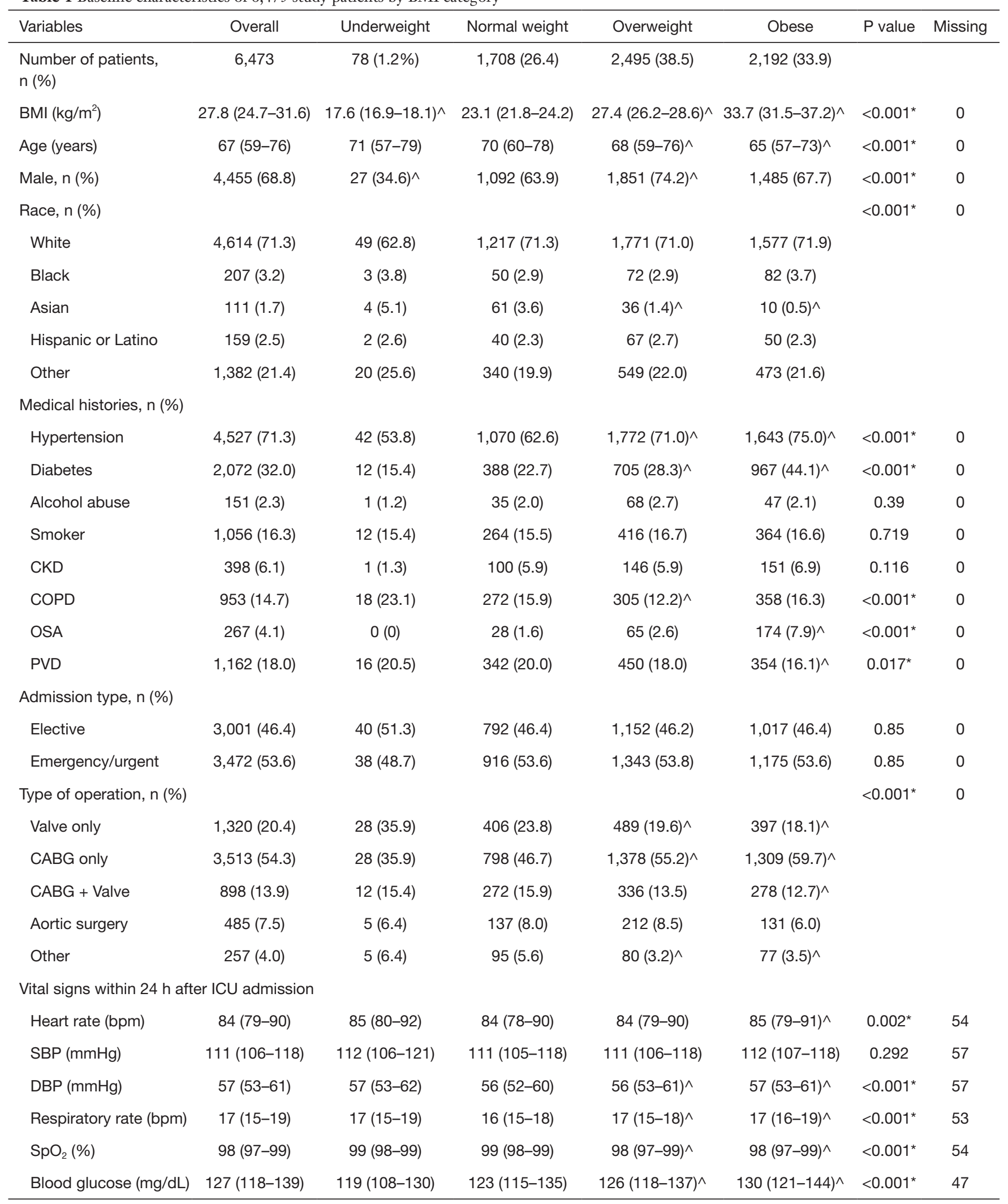

Table 1 (continued) 
Table 1 (continued)

\begin{tabular}{|c|c|c|c|c|c|c|c|}
\hline Variables & Overall & Underweight & Normal weight & Overweight & Obese & $P$ value & Missing \\
\hline $\mathrm{sCr}(\mathrm{mg} / \mathrm{dL})$ & $0.9(0.7-1.1)$ & $0.7(0.6-1.0)^{\wedge}$ & $0.8(0.7-1.0)$ & $0.9(0.7-1.1)^{\wedge}$ & $0.9(0.8-1.1)^{\wedge}$ & $<0.001^{*}$ & 30 \\
\hline WBC $\left(10^{9} / L\right)$ & $12.1(9.7-15.1)$ & $10.6(8.5-14.3)$ & $11.5(9.3-14.6)$ & $11.9(9.6-14.8)$ & $12.7(10.2-15.7)^{\wedge}$ & $<0.001^{*}$ & 129 \\
\hline Hemoglobin (g/dL) & $10.4(9.5-11.5)$ & $9.7(9.0-10.2)^{\wedge}$ & $10.1(9.3-11.0)$ & $10.5(9.5-11.5)^{\wedge}$ & $10.6(9.6-11.7)^{\wedge}$ & $<0.001^{*}$ & 16 \\
\hline PT (s) & $14.8(14.0-15.8)$ & $15.2(14.2-16.3)$ & $15.0(14.2-15.9)$ & $14.8(14.0-15.8)^{\wedge}$ & $14.7(13.9-15.6)^{\wedge}$ & $<0.001^{*}$ & 267 \\
\hline APTT (s) & 35.6 (30.9-43.6) & 39.7 (33.9-51.9) & $38.3(33.3-47.3)$ & $35.8(31.1-43.7)^{\wedge}$ & $33.2(29.5-39.5)^{\wedge}$ & $<0.001^{*}$ & 262 \\
\hline $\begin{array}{l}\text { SAPS II score after ICU } \\
\text { admission }\end{array}$ & $33(27-41)$ & 31 (25-39) & $34(27-42)$ & $33(27-40)$ & $33(26-41)^{\wedge}$ & $0.013^{\star}$ & 0 \\
\hline
\end{tabular}

BMI, body mass index; CKD, chronic kidney disease; COPD, chronic obstructive pulmonary disease; OSA, obstructive sleep apnea; PVD, peripheral vascular disease; CABG, coronary artery bypass grafting; SBP, systolic blood pressure; DBP, diastolic blood pressure; sCr, serum creatinine; WBC, white blood cell count; SAPS-II, Simplified Acute Physiology Score II. Data are represented as median (interquartile range) or $\mathrm{n}(\%) .{ }^{*}$, indicate statistical significance. $\wedge$, significant difference compared with normal weight category.

Table 2 Univariate analysis of clinical outcomes in 6,473 patients by BMI category

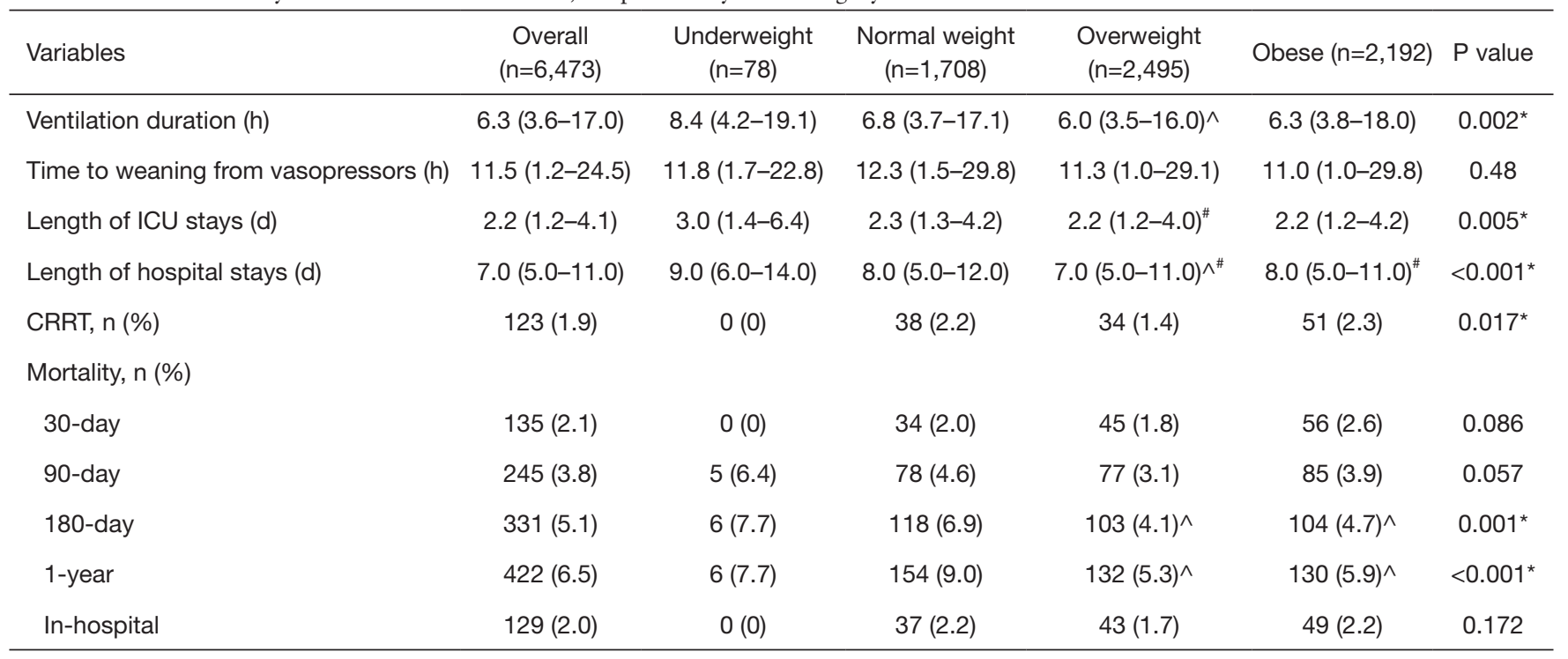

CRRT, continuous renal replacement therapy. Data are represented as median (interquartile range) or $\mathrm{n}(\%) .{ }^{*}$, indicate statistical significance. ^, significant difference compared with normal weight category; ${ }^{*}$, significant difference compared with underweight category.

patients $(\mathrm{P}<0.001)$. Obese patients had a higher heart rate $(\mathrm{P}=0.002)$, WBC count $(\mathrm{P}<0.001)$, platelet count $(\mathrm{P}<0.001)$, and lower SAPS II score $(\mathrm{P}=0.013)$ compared with normal weight patients.

\section{Study outcomes}

Comparisons of the primary and secondary clinical outcomes among the four BMI categories are shown in Table 2. Ventilation duration (median time, 6.0 vs. 6.8 hours, $\mathrm{P}=0.002$ ) and length of hospital stay (median time, 7.0 vs. 8.0 days, $\mathrm{P}<0.001$ ) were shorter in the overweight group compared to the normal weight group. Length of ICU stay was shorter in the overweight group compared to the underweight group (median time, 2.2 vs. 3.0 days, $\mathrm{P}=0.005$ ). The 1-year mortality rates were lower in the overweight 


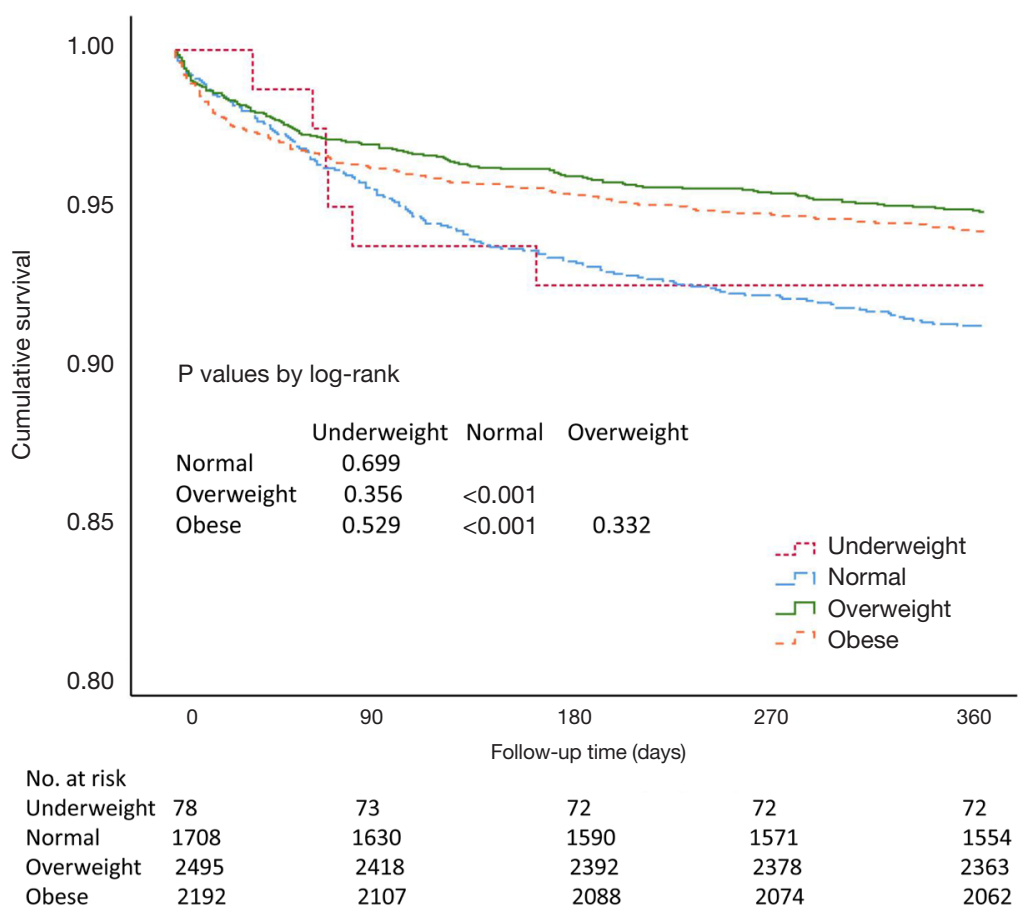

Figure 2 Kaplan-Meier curves of 1-year mortality by BMI category.

(5.3\%) and obese (5.9\%) group compared with the normal weight group (9.0\%).

The Kaplan-Meier survival curve of 1-year mortality is shown in Figure 2, and suggests the notable 1-year survival advantage in the overweight and obese group compared with their normal weight counterparts (log-rank test $\mathrm{P}<0.001)$.

Multivariate Cox proportional hazard analysis showed that, overweight patients had a lower 1-year mortality compared to those with normal weight ( $\mathrm{HR}=0.660,95 \%$ CI: $0.516-0.843, \mathrm{P}=0.001$, Table 3). In addition, age, hypertension, smoking, COPD, peripheral vascular disease, type of admission, type of operation, $\mathrm{SpO}_{2}$, blood glucose, serum creatinine, WBC, hemoglobin, PT, APTT and SAPS II score were also independently linked to 1-year mortality with a $\mathrm{P}<0.05$ (Table 3). Multivariate logistic regression analysis showed that BMI category was not independently associated with in-hospital mortality $(\mathrm{P}=0.073$, Table 3). Age, hypertension, smoker, alcohol abuse, systolic blood pressure, $\mathrm{SpO}_{2}$, blood glucose, serum creatinine, APTT and SAPS II score were independently associated with inhospital mortality with a $\mathrm{P}<0.05$ (Table 3).

BMI was also analyzed as a continuous variable. The BMI cutoff of $30 \mathrm{~kg} / \mathrm{m}^{2}$, was determined based on the shape of the restricted cubic splines (Figure 3). Multivariate Cox proportional hazard analysis showed that, in the BMI $<30 \mathrm{~kg} / \mathrm{m}^{2}$ subgroup, each $1 \mathrm{~kg} / \mathrm{m}^{2}$ of BMI increase was independently associated with a significant decrease in the 1 -year mortality risk (HR $=0.936,95 \%$ CI: 0.899-0.975, $\mathrm{P}=0.002$, Table 4), while this association was not present in the BMI $\geq 30 \mathrm{~kg} / \mathrm{m}^{2}$ subgroup (HR $=1.032,95 \%$ CI: 0.998 1.067, $\mathrm{P}=0.064$, Table 4).

\section{Subgroup analysis}

Subgroup analysis was performed according to age, hypertension and types of surgery (Figure 4). In the age 6080 and $>80$ years subgroups, overweight was significantly associated with a lower 1-year mortality compared with normal/underweight patients (HR $=0.651,95 \%$ CI: 0.483 0.879 and HR $=0.465,95 \%$ CI: $0.294-0.737$, respectively), while in patients aged $\leq 60$ years, the relationship was not significant $(\mathrm{P}=0.480)$. In patients with hypertension, overweight or obese was significantly associated with a lower 1-year mortality compared with normal/underweight patients (HR $=0.504,95 \% \mathrm{CI}: 0.375-0.677$ and HR $=0.566,95 \%$ CI: $0.423-0.759$, respectively), while in nonhypertensive patients, this relationship was not significant 
Table 3 Multivariate logistic regression analysis for in-hospital mortality and multivariate Cox proportional hazard analysis for 1-year mortality

\begin{tabular}{|c|c|c|c|c|c|c|}
\hline Variables & \multicolumn{3}{|c|}{ In-hospital mortality } & \multicolumn{3}{|c|}{ 1-year mortality } \\
\hline BMI category & & & 0.073 & & & $0.009^{*}$ \\
\hline Underweight vs. normal & & Not estimable & 0.997 & 0.633 & $0.256-1.566$ & 0.323 \\
\hline Overweight vs. normal & 0.856 & $0.502-1.460$ & 0.568 & 0.66 & $0.516-0.843$ & $0.001^{*}$ \\
\hline Age (years) & 1.026 & $1.003-1.050$ & $0.024^{*}$ & 1.035 & $1.023-1.047$ & $<0.001^{*}$ \\
\hline Male & 0.713 & $0.450-1.130$ & 0.15 & 0.977 & $0.780-1.224$ & 0.841 \\
\hline Race (ref. White) & & & 0.74 & & & 0.426 \\
\hline Black & 0.911 & $0.295-2.807$ & 0.871 & 1.208 & $0.713-2.047$ & 0.483 \\
\hline Other & 1.124 & $0.677-1.867$ & 0.652 & 1.151 & $0.899-1.473$ & 0.265 \\
\hline Hypertension & 0.476 & $0.304-0.745$ & $0.001^{*}$ & 0.626 & $0.502-0.781$ & $<0.001^{*}$ \\
\hline Diabetes & 0.932 & $0.573-1.517$ & 0.778 & 1.161 & $0.918-1.469$ & 0.213 \\
\hline Alcohol abuse & 3.285 & $1.159-9.308$ & $0.025^{\star}$ & 1.364 & $0.696-2.674$ & 0.366 \\
\hline Smoker & 0.269 & $0.110-0.655$ & $0.004^{*}$ & 0.719 & $0.521-0.992$ & $0.045^{\star}$ \\
\hline CKD & 1.47 & $0.758-2.849$ & 0.254 & 1.33 & $0.957-1.849$ & 0.089 \\
\hline COPD & 1.255 & $0.727-2.166$ & 0.414 & 1.594 & $1.244-2.042$ & $<0.001^{*}$ \\
\hline OSA & 1.189 & $0.445-3.176$ & 0.73 & 0.899 & $0.519-1.556$ & 0.703 \\
\hline Aortic surgery & 2.342 & 0.999-5.490 & 0.05 & 1.39 & $0.903-2.140$ & 0.135 \\
\hline Other & 2.313 & $0.838-6.386$ & 0.106 & 1.994 & $1.203-3.307$ & $0.007^{\star}$ \\
\hline Heart rate (bpm) & 0.99 & $0.971-1.010$ & 0.331 & 0.999 & $0.990-1.007$ & 0.919 \\
\hline $\mathrm{SBP}(\mathrm{mmHg})$ & 0.977 & $0.955-1.000$ & $0.046^{\star}$ & 0.996 & $0.984-1.007$ & 0.456 \\
\hline $\mathrm{DBP}(\mathrm{mmHg})$ & 0.986 & $0.956-1.017$ & 0.387 & 1.004 & $0.989-1.020$ & 0.576 \\
\hline Respiratory rate (bpm) & 1.061 & $0.995-1.130$ & $0.07^{\star}$ & 1.033 & $0.999-1.069$ & 0.057 \\
\hline $\mathrm{SpO}_{2}(\%)$ & 0.854 & $0.766-0.951$ & $0.004^{*}$ & 0.925 & $0.887-0.965$ & $<0.001^{*}$ \\
\hline Blood glucose (mg/dL) & 1.014 & $1.007-1.020$ & $<0.001^{\star}$ & 1.008 & $1.005-1.012$ & $<0.001^{*}$ \\
\hline $\mathrm{sCr}(\mathrm{mg} / \mathrm{dL})$ & 1.481 & $1.312-1.673$ & $<0.001^{*}$ & 1.291 & $1.217-1.369$ & $<0.001^{*}$ \\
\hline WBC $\left(10^{9} / \mathrm{L}\right)$ & 1.022 & 0.986-1.059 & 0.239 & 1.02 & $1.004-1.036$ & $0.014^{\star}$ \\
\hline Hemoglobin (g/dL) & 0.951 & $0.804-1.123$ & 0.551 & 0.862 & $0.792-0.939$ & $0.001^{*}$ \\
\hline
\end{tabular}

Table 3 (continued) 
Table 3 (continued)

\begin{tabular}{|c|c|c|c|c|c|c|}
\hline Variables & \multicolumn{3}{|c|}{ In-hospital mortality } & \multicolumn{3}{|c|}{ 1-year mortality } \\
\hline Platelet $\left(10^{9} / \mathrm{L}\right)$ & 1.001 & $0.998-1.004$ & 0.522 & 1.001 & $0.999-1.002$ & 0.289 \\
\hline PT (s) & 1.006 & $0.956-1.058$ & 0.829 & 1.028 & $1.005-1.051$ & $0.016^{*}$ \\
\hline APTT (s) & 1.036 & $1.025-1.046$ & $<0.001^{\star}$ & 1.021 & $1.015-1.026$ & $<0.001^{*}$ \\
\hline
\end{tabular}

BMI, body mass index; CKD, chronic kidney disease; COPD, chronic obstructive pulmonary disease; OSA, obstructive sleep apnea; PVD, peripheral vascular disease; CABG, coronary artery bypass grafting; SBP, systolic blood pressure; DBP, diastolic blood pressure; sCr, serum creatinine; WBC, white blood cell count; SAPS-II, Simplified Acute Physiology Score II. *, indicate statistical significance.

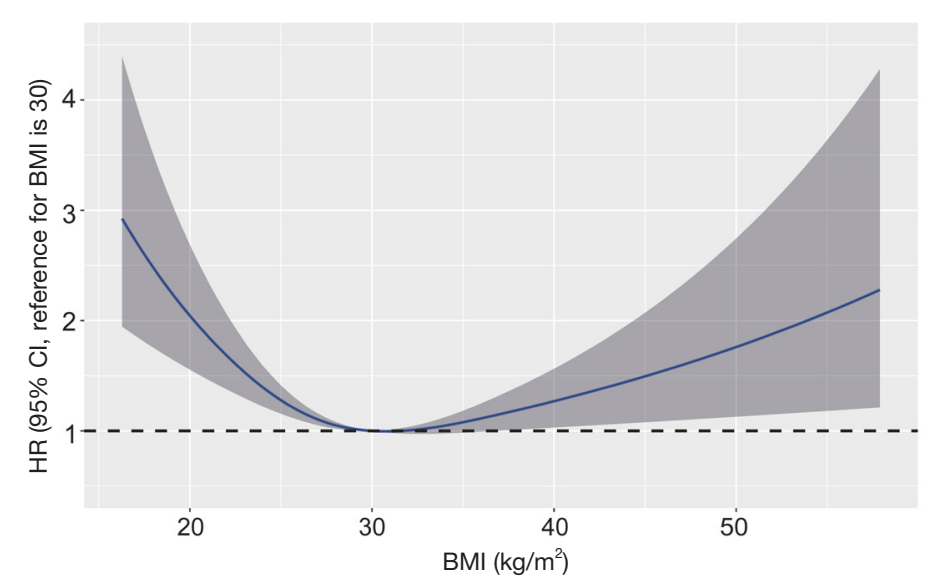

Figure 3 Association of BMI and 1-year mortality (using restricted cubic splines with 3 knots). HR, hazard ratio.

$(\mathrm{P}=0.391)$. Of the surgery type subgroups (valve only, CABG only, CABG + valve and aortic), only in the CABG subgroup was overweight or obese significantly associated with a lower 1-year mortality compared with normal/ underweight patients (HR $=0.483,95 \%$ CI: $0.333-0.703$ and HR $=0.658,95 \%$ CI: 0.463-0.934, respectively).

\section{Case-control matched analysis}

Overweight and obese patients were separately matched 1:1 with normal/underweight patients based on age ( \pm 2 years) and sex. Kaplan-Meier survival curves of 1 -year mortality in matched cohorts are shown in Figure 5, and indicated that 1 -year survival is still higher in the overweight and obese groups compared with the normal/underweight group (logrank test $\mathrm{P}<0.001)$. Subgroup analysis was also performed in the matched cohorts (Table 5), and suggested that age and sex differences did not affect the survival advantage of the overweight and obese groups in the hypertension and isolated CABG cohorts.

\section{Discussion}

The present study suggested that overweight patients undergoing cardiac surgery with extracorporeal circulation had better 1-year survival compared to their normal weight counterparts after adjusting for confounding factors. The optimal BMI for 1-year survival after cardiac surgery appeared to approximate the overweight/mild obesity level $\left(\mathrm{BMI}=30 \mathrm{~kg} / \mathrm{m}^{2}\right)$. This obesity paradox was limited to patients with advanced age ( $>60$ years), hypertension, and those undergoing isolated CABG.

Studies of the relationship between BMI and cardiac surgery outcome in diverse patient populations and with different follow-up times have generated controversial results. A nationwide cohort study comprising 401,227 
Table 4 Multivariate Cox proportional hazard analysis for 1-year mortality in BMI subgroups (BMI analyzed as a continuous variable)

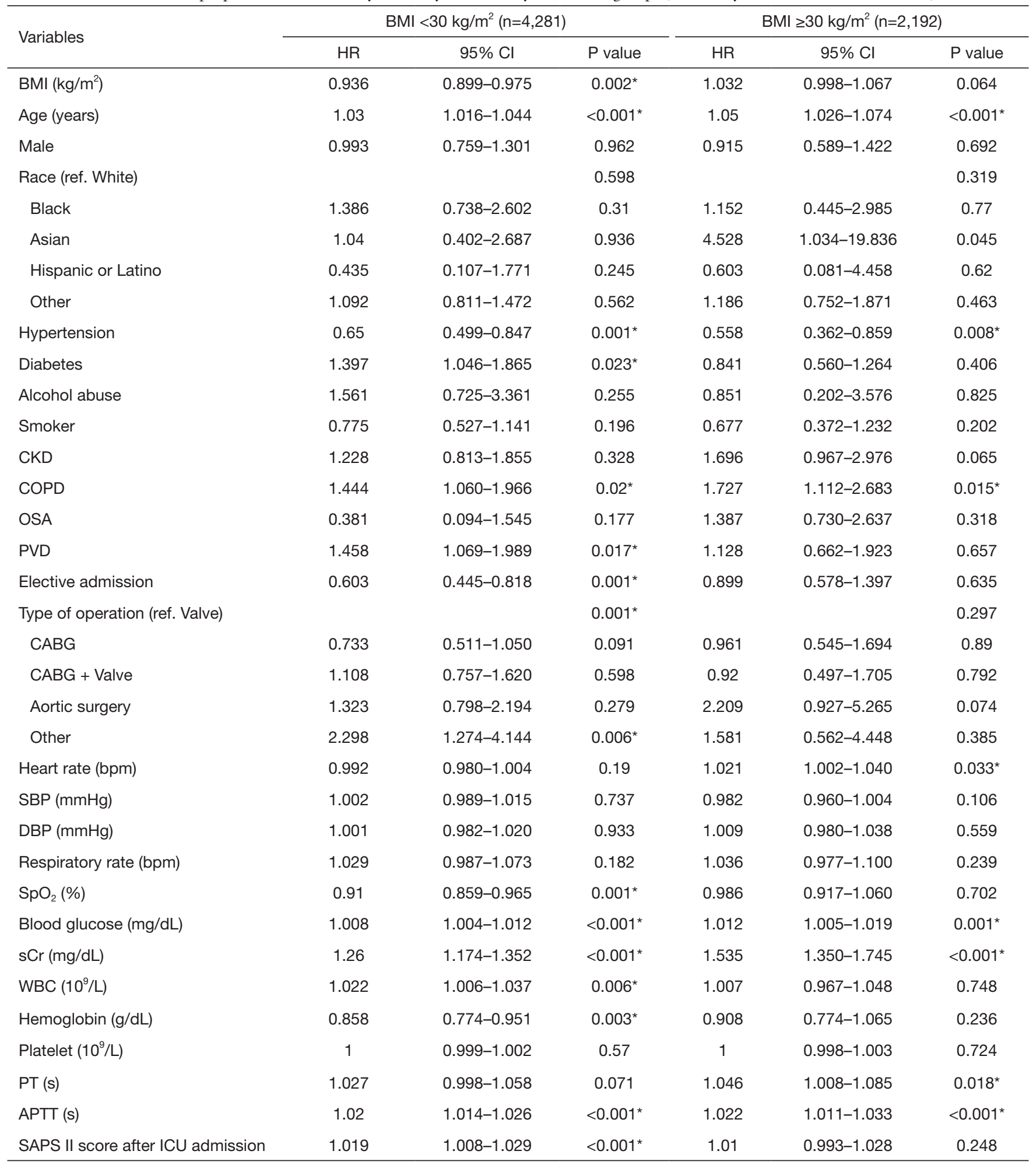

BMI, body mass index; CKD, chronic kidney disease; COPD, chronic obstructive pulmonary disease; OSA, obstructive sleep apnea; PVD, peripheral vascular disease; CABG, coronary artery bypass grafting; SBP, systolic blood pressure; DBP, diastolic blood pressure; sCr, serum creatinine; WBC, white blood cell count; SAPS-II, Simplified Acute Physiology Score II. *, indicate statistical significance. 


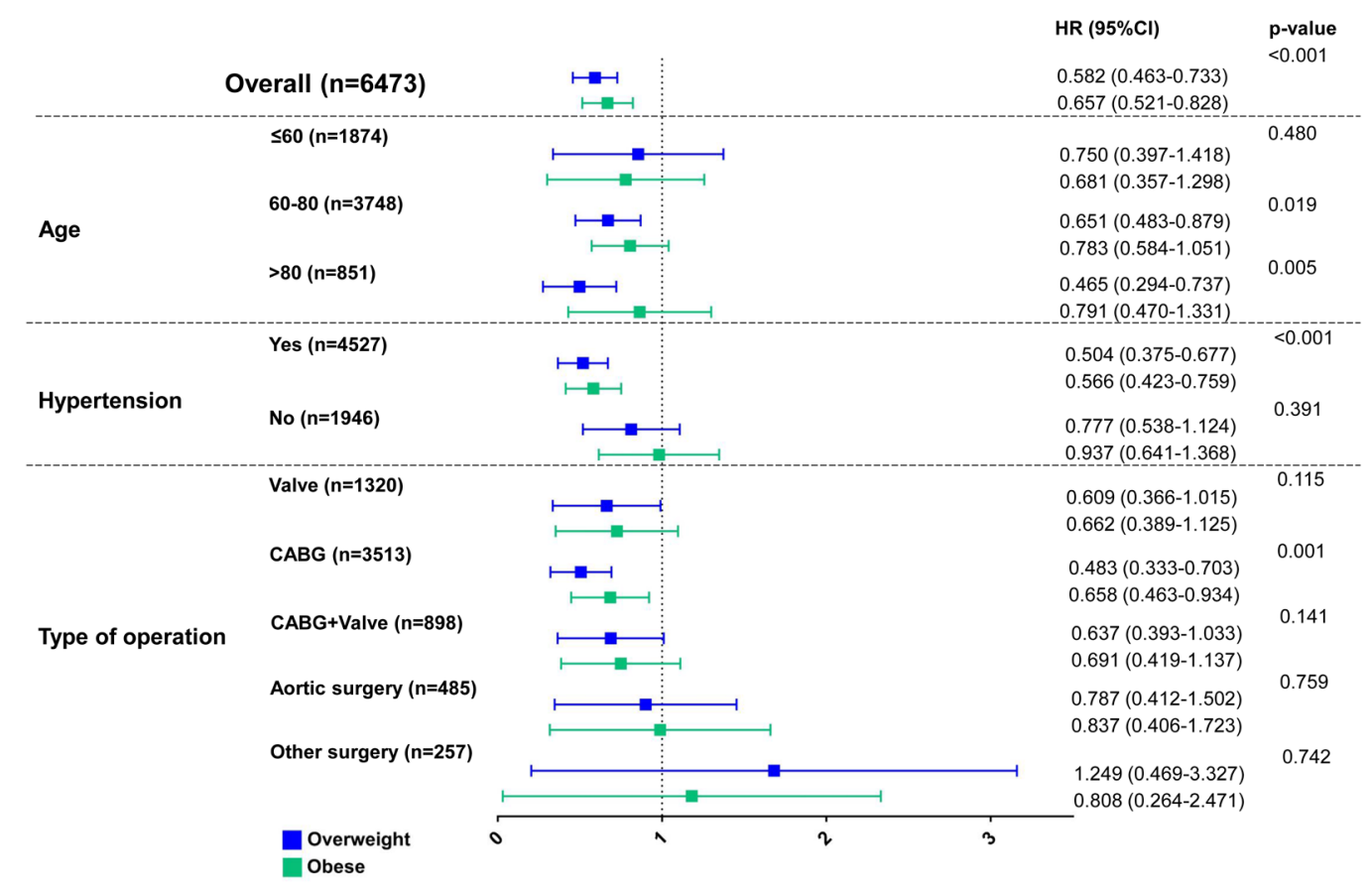

Figure 4 Hazard ratios of 1-year mortality risk on the stratification of age levels, hypertension and types of operation. Patients with BMI $<25 \mathrm{~kg} / \mathrm{m}^{2}$ (underweight and normal weight) acts as the reference group.

patients undergoing cardiac surgery observed a decreased in-hospital mortality in overweight and obese class I and II patients, and an increased in-hospital mortality in underweight patients (6). Another large cohort study including 96,058 elderly patients ( $\geq 60$ years old) receiving isolated CABG showed that low $\left(<18.5 \mathrm{~kg} / \mathrm{m}^{2}\right)$ and high $\left(\geq 30 \mathrm{~kg} / \mathrm{m}^{2}\right)$ BMI were associated with higher risk of operative mortality (13). However, a recent study of 16,252 patients undergoing mitral valve surgery for degenerative disease failed to demonstrate an association between BMI and in-hospital outcome (8). Study results pertaining to long-term outcomes, are also inconsistent. One study comprising 78,762 patients receiving CABG or combined $\mathrm{CABG}$ /aortic valve replacement showed that overweight and obese patients had better 1- and 5-year survival rates (14), while others found no relationship between high BMI and an increased probability of long-term survival $(9,15)$. The results of the present study agreed with previous reports that reported overweight to be associated with better longterm post-cardiac surgery survival compared to normal weight (16).

There may be several reasons explaining the obesity paradox seen in cardiac surgery. Obese patients are usually younger, while underweight patients are more likely to have frailty, cachexia, or severe chronic disease, which may lead to a confounding bias (11). Another hypothesis is that obese patients are admitted to cardiac surgery only if they are subjectively at lower risk, leading to a selection bias (15). In the present study, overweight remained an independent protective factor for 1-year survival after adjusting for confounding factors, which suggested that there may be potential mechanisms underlying the direct protective effect of a higher BMI. Some adipokines produced by adipose tissue had been proved to provide cardiovascular benefits, such as regeneration of damaged myocardium, reduction of afterload, inhibition of myocardium fibrosis, and promotion of angiogenesis (17).

The present study showed that the protective effect of overweight after cardiac surgery was confined to elderly patients ( $>60$ years). Similar findings have been observed in patients with ST-segment elevation myocardial infarction (18), atrial fibrillation (19), and in survivors of sudden cardiac arrest (20). Many age-related changes including increases in visceral fatness, chronic inflammation, oxidative stress, decreased body height, muscle mass, energy expenditure, changes in neurohormonal, metabolism, and 

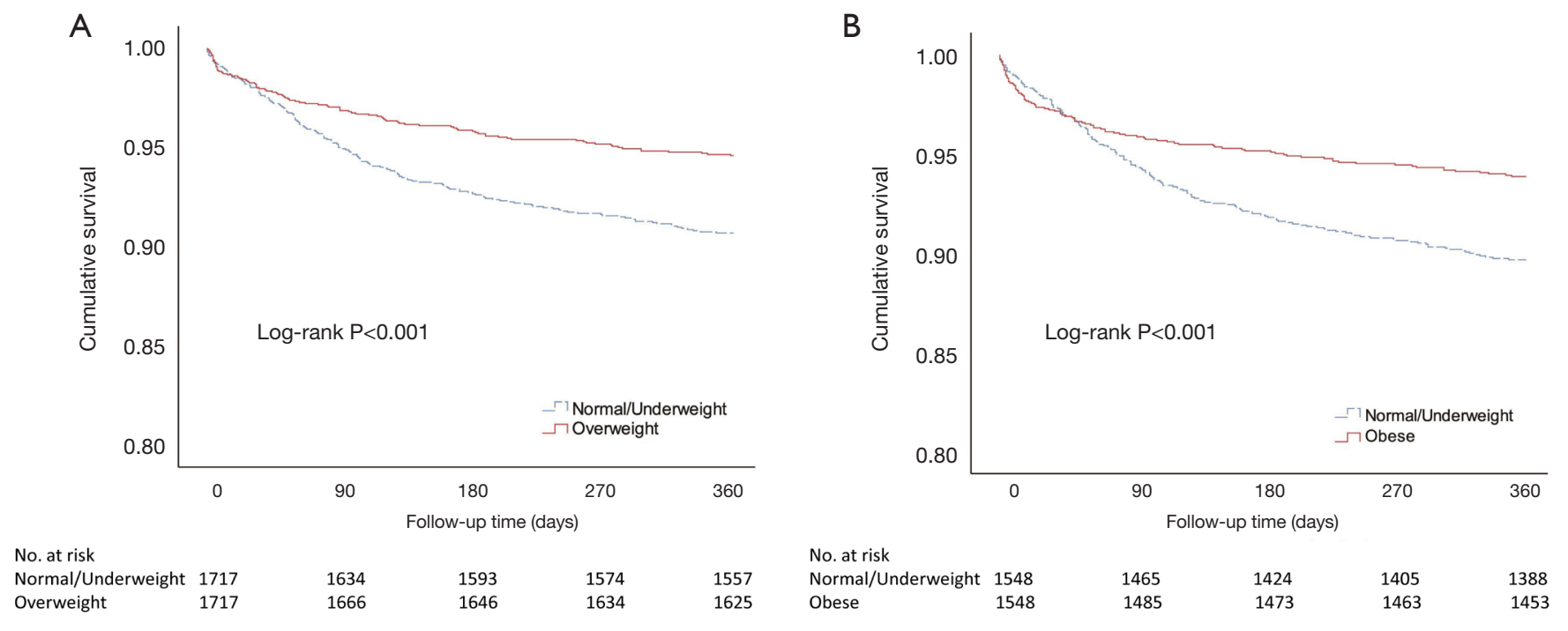

Figure 5 Kaplan-Meier curves of 1-year mortality in age and sex matched cohorts. Overweight (A) and obese (B) patients were separately matched 1:1 with normal/underweight patients based on age ( \pm 2 years) and sex.

Table 5 Subgroup analysis in age and sex matched cohorts

\begin{tabular}{|c|c|c|c|c|}
\hline Study cohort & Sample size & HR & $95 \% \mathrm{Cl}$ & $P$ value \\
\hline \multicolumn{5}{|l|}{$\begin{array}{l}\text { Overweight } \\
\text { vs. Normal/ } \\
\text { underweight } \\
\text { matched }\end{array}$} \\
\hline Overall & 3,434 & 0.571 & $0.443-0.738$ & $<0.001^{*}$ \\
\hline Hypertension & 2,293 & 0.463 & $0.330-0.650$ & $<0.001^{*}$ \\
\hline Isolated CABG & 1,706 & 0.546 & $0.363-0.822$ & $0.004^{*}$ \\
\hline \multicolumn{5}{|l|}{$\begin{array}{l}\text { Obese vs. normal/ } \\
\text { underweight } \\
\text { matched }\end{array}$} \\
\hline Overall & 3,096 & 0.586 & $0.454-0.755$ & $<0.001^{*}$ \\
\hline Hypertension & 2,115 & 0.485 & $0.350-0.670$ & $<0.001^{*}$ \\
\hline Isolated CABG & 1,631 & 0.549 & $0.368-0.817$ & $0.003^{*}$ \\
\hline
\end{tabular}

CABG, coronary artery bypass grafting. *, indicate statistical significance.

higher prevalence of frailty, undernutrition and chronic disease, might contribute to this phenomenon (21). Another interesting finding of our subgroup analysis was that overweight/obese served as a protective factor for postcardiac surgery survival only in hypertensive patients. Previous studies have shown that in a population with hypertension and coronary artery disease, overweight and obese patients had decreased morbidity and mortality compared with normal weight patients (22). A remarkable disparity in regional sympathetic activity between normal weight hypertension and obesity-hypertension has also been reported. Cardiac sympathetic activity was increased in normal weight hypertensive patients, but was at a normal or low level in those who were obese (23). The theory that a more toxic form of sympathetic activation may be present in normal weight hypertension compared with obese-hypertension might explain the obesity paradox in hypertension. Our subgroup analysis also suggested that the association between BMI and 1-year survival was found only in isolated CABG patients, which was consistent with previous cohort studies and meta-analyses $(16,24)$. However, a recent meta-analysis presented the opposite results, in which a decreased mortality was found in patients after valve surgery but not after CABG (7). Further studies are needed to determine the prognostic effect of $\mathrm{BMI}$ in different types of cardiac surgery.

The limitations of the present study are as follows: first, this is a retrospective single-center study, and it was difficult to exclude the influence of some potential confounding factors on the results. Secondly, due to the nature of the MIMIC database, some important information such as the New York Heart Association functional class, cardiac function parameters and operative details were not available. 
Moreover, the sample size of some subgroups was small, and might not have provided sufficient statistical power to draw credible conclusions.

\section{Conclusions}

In conclusion, the present retrospective cohort study showed that overweight was associated with better 1-year survival in patients after cardiac surgery when compared to normal weight. The protective effect of overweight on postcardiac surgery survival was confined to elderly patients (>60 years).

\section{Acknowledgments}

The datasets analyzed in the present study are available in the MIMIC-III v1.4 (https://mimic.physionet.org). We appreciate the efforts of the Medical Information Mart for Intensive Care III Database. Thanks to the researchers at the MIT Laboratory for Computational Physiology and collaborating research groups.

Funding: This work was supported by grant from Natural Science Foundation of Zhejiang Province, China (Grant No. LQ20H020003, Grant No. LY20H020003), National Natural Science Foundation of China (Grant No. 81670350), and Key Research and Development Program of Zhejiang Province, China (Grant No. 2019C03008), Health \& Medicine Science and Technology Program of Zhejiang Province, China (Grant No. 2019KY377).

\section{Footnote}

Reporting Checklist: The authors have completed the STROBE reporting checklist. Available at http://dx.doi. org/10.21037/jtd-20-2824

Conflicts of Interest: All authors have completed the ICMJE uniform disclosure form (available at http://dx.doi. org/10.21037/jtd-20-2824). The authors have no conflicts of interest to declare.

Ethical Statement: The authors are accountable for all aspects of the work in ensuring that questions related to the accuracy or integrity of any part of the work are appropriately investigated and resolved. The study was conducted in accordance with the Declaration of Helsinki (as revised in 2013).
Open Access Statement: This is an Open Access article distributed in accordance with the Creative Commons Attribution-NonCommercial-NoDerivs 4.0 International License (CC BY-NC-ND 4.0), which permits the noncommercial replication and distribution of the article with the strict proviso that no changes or edits are made and the original work is properly cited (including links to both the formal publication through the relevant DOI and the license). See: https://creativecommons.org/licenses/by-nc-nd/4.0/.

\section{References}

1. Finucane MM, Stevens GA, Cowan MJ, et al. National, regional, and global trends in body-mass index since 1980: systematic analysis of health examination surveys and epidemiological studies with 960 country-years and 9.1 million participants. Lancet 2011;377:557-67.

2. Lavie CJ, McAuley PA, Church TS, et al. Obesity and cardiovascular diseases: implications regarding fitness, fatness, and severity in the obesity paradox. J Am Coll Cardiol 2014;63:1345-54.

3. Flegal KM, Kit BK, Orpana H, et al. Association of allcause mortality with overweight and obesity using standard body mass index categories: a systematic review and metaanalysis. JAMA 2013;309:71-82.

4. Niedziela J, Hudzik B, Niedziela N, et al. The obesity paradox in acute coronary syndrome: a meta-analysis. Eur J Epidemiol 2014;29:801-12.

5. Padwal R, McAlister FA, McMurray JJ, et al. The obesity paradox in heart failure patients with preserved versus reduced ejection fraction: a meta-analysis of individual patient data. Int J Obes (Lond) 2014;38:1110-4.

6. Mariscalco G, Wozniak MJ, Dawson AG, et al. Body Mass Index and Mortality Among Adults Undergoing Cardiac Surgery: A Nationwide Study With a Systematic Review and Meta-Analysis. Circulation 2017;135:850-63.

7. Liu X, Xie L, Zhu W, et al. Association of body mass index and all-cause mortality in patients after cardiac surgery: A dose-response meta-analysis. Nutrition 2020;72:110696.

8. Burns DJP, Rapetto F, Angelini GD, et al. Body mass index and early outcomes following mitral valve surgery for degenerative disease. J Cardiothorac Vasc Anesth 2021;35:492-8.

9. Burgos LM, Gil Ramirez A, Seoane L, et al. Is the Obesity Paradox in Cardiac Surgery Really a Myth? Effect of Body Mass Index on Early and Late Clinical Outcomes. J Cardiothorac Vasc Anesth 2020. [Epub ahead of print]. 10. Dixon JB, Lambert GW. The obesity paradox--a reality 
that requires explanation and clinical interpretation. Atherosclerosis 2013;226:47-8.

11. Preston SH, Stokes A. Obesity paradox: conditioning on disease enhances biases in estimating the mortality risks of obesity. Epidemiology 2014;25:454-61.

12. Johnson AE, Pollard TJ, Shen L, et al. MIMIC-III, a freely accessible critical care database. Sci Data 2016;3:160035.

13. Nishioka N, Ichihara N, Bando K, et al. Body mass index as a tool for optimizing surgical care in coronary artery bypass grafting through understanding risks of specific complications. J Thorac Cardiovasc Surg 2020;160:409420.e14.

14. Johnson AP, Parlow JL, Whitehead M, et al. Body Mass Index, Outcomes, and Mortality Following Cardiac Surgery in Ontario, Canada. J Am Heart Assoc 2015;4:e002140.

15. Ho KM, Bertenshaw C, Same S, et al. Differential associations between body mass index and outcomes after elective adult cardiac surgery: a linked data cohort study. Anaesth Intensive Care 2013;41:573-83.

16. Takagi H, Umemoto T, Group A. Overweight, but not obesity, paradox on mortality following coronary artery bypass grafting. J Cardiol 2016;68:215-21.

17. Sawicka M, Janowska J, Chudek J. Potential beneficial effect of some adipokines positively correlated with the adipose tissue content on the cardiovascular system. Int J Cardiol 2016;222:581-9.

Cite this article as: Zhang Y, Zheng Q, Dai X, Xu X, Ma L. Overweight is associated with better one-year survival in elderly patients after cardiac surgery: a retrospective analysis of the MIMICIII database. J Thorac Dis 2021;13(2):562-574. doi: 10.21037/jtd-202824
18. Neeland IJ, Das SR, Simon DN, et al. The obesity paradox, extreme obesity, and long-term outcomes in older adults with ST-segment elevation myocardial infarction: results from the NCDR. Eur Heart J Qual Care Clin Outcomes 2017;3:183-91.

19. Yanagisawa S, Inden $Y$, Yoshida $N$, et al. Body mass index is associated with prognosis in Japanese elderly patients with atrial fibrillation: an observational study from the outpatient clinic. Heart Vessels 2016;31:1553-61.

20. Matinrazm S, Ladejobi A, Pasupula DK, et al. Effect of body mass index on survival after sudden cardiac arrest. Clin Cardiol 2018;41:46-50.

21. Wang S, Ren J. Obesity Paradox in Aging: From Prevalence to Pathophysiology. Prog Cardiovasc Dis 2018;61:182-9.

22. Uretsky S, Messerli FH, Bangalore S, et al. Obesity paradox in patients with hypertension and coronary artery disease. Am J Med 2007;120:863-70.

23. Esler M, Lambert G, Schlaich M, et al. Obesity Paradox in Hypertension: Is This Because Sympathetic Activation in Obesity-Hypertension Takes a Benign Form? Hypertension 2018;71:22-33.

24. Zittermann A, Becker T, Gummert JF, et al. Body mass index, cardiac surgery and clinical outcome. A singlecenter experience with 9125 patients. Nutr Metab Cardiovasc Dis 2014;24:168-75. 